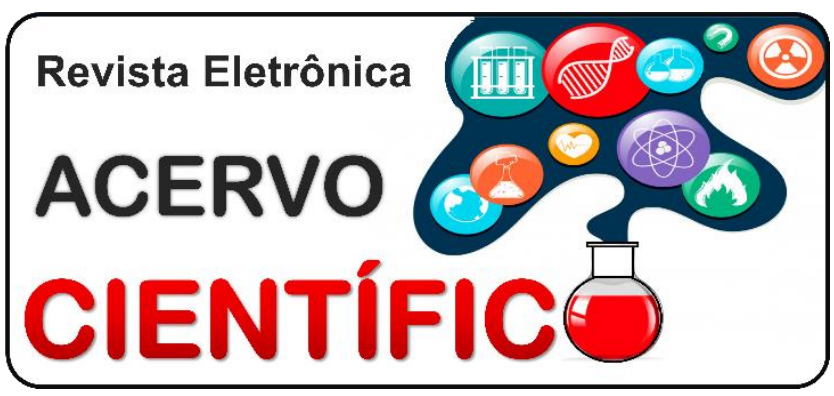

\section{RELATO DE EXPERIÊNCIA}

Recebido em: 1/2020

Aceito em: 2/2020

Publicado em: 9/2020

\title{
A importância do processo de educação em saúde entre estudantes da área da saúde: um relato de experiência
}

\author{
The importance of the health education process among health students: an experience \\ report
}

La importancia del proceso de educación en salud entre estudiantes de salud: un informe de experiência

Adilson Mendes de Figueiredo Júnior ${ }^{1 *}$, Janice de Matos Frazão ${ }^{1}$, Amanda Thaís Silva da Silva ${ }^{1}$, Lucas Monteiro da Trindade ${ }^{1}$, Thaís Maria de Souza Contente ${ }^{1}$, Thayse Hage Gomes Machado ${ }^{1}$, Clenilde dos Santos Fernandes ${ }^{1}$, Deisiane do Socorro da Silva Barbosa ${ }^{1}$, Clebson Luis Trindade da Silva ${ }^{1}$, Ana Carla de Sousa Aguiar².

\begin{abstract}
Resumo: Esse artigo buscou relatar a experiência de alunos do $5^{\circ}$ semestre do curso de graduação em Enfermagem, Fisioterapia e Nutrição sobre a importância do processo de educação em saúde como forma de valorização da saúde, através de um estudo descritivo, tipo relato de experiência, elaborado no contexto do estágio extracurricular em uma unidade básica de saúde. A educação em saúde pode ser considerada uma das principais ações de promoção da saúde, já que se revela de suma importância tanto na prevenção e reabilitação de doenças, além de despertar a cidadania, responsabilidade pessoal e social, bem como a formação de multiplicadores e cuidadores. Desta forma, a educação em saúde tornou-se um processo complexo que, ao unir um conjunto de saberes e práticas diversas, busca proporcionar às pessoas o mais alto nível de saúde. Esse conceito, entretanto, é fruto de um processo lento que vem ao longo dos anos acompanhando as mudanças políticas e econômicas no Brasil.
\end{abstract}

Palavras-chave: Educação em saúde, Saúde pública, Tecnologia em saúde.

\begin{abstract}
This article sought to report the experience of students from the 5th semester of the undergraduate course in Nursing. Physiotherapy and Nutrition on the importance of the health education process as a way of valuing health, through a descriptive study, type of experience report, elaborated in the context of the extracurricular internship in a basic health unit. Health education can be considered one of the main health promotion actions, since it is of paramount importance both in the prevention and rehabilitation of diseases, in addition to awakening citizenship, personal and social responsibility, as well as the formation of multipliers and caregivers. . In this way, health education has become a complex process that, by uniting a set of diverse knowledge and practices, seeks to provide people with the highest level of health. This concept, however, is the result of a slow process that has followed the political and economic changes in Brazil over the years.
\end{abstract}

Keywords: Health education, Public health, Health technology.

\footnotetext{
${ }^{1}$ Escola Superior da Amazônia (ESAMAZ), Belém - Pará. *E-mail: adilsonmdfj@hotmail.com
}

2 Universidade do Estado do Pará (UEPA). Belém - Pará. 
Resumen: Este artículo buscó informar la experiencia de los estudiantes del quinto semestre del curso de pregrado en Enfermería, Fisioterapia y Nutrición sobre la importancia del proceso de educación en salud como una forma de valorar la salud, a través de un estudio descriptivo, tipo de informe de experiencia, desarrollado en el contexto de la pasantía extracurricular en una unidad básica de salud. La educación para la salud puede considerarse una de las principales acciones de promoción de la salud, ya que es de suma importancia tanto en la prevención y rehabilitación de enfermedades, además de despertar la ciudadanía, la responsabilidad personal y social, como la formación de multiplicadores y cuidadores. De esta manera, la educación para la salud se ha convertido en un proceso complejo que, al unir un conjunto de conocimientos y prácticas diversas, busca proporcionar a las personas el más alto nivel de salud. Sin embargo, este concepto es el resultado de un proceso lento que ha seguido los cambios políticos y económicos en Brasil a lo largo de los años.

Palabras clave: Educación para la salud, Salud pública, Tecnología de salud.

\section{INTRODUÇÃO}

A educação em saúde, conhecida inicialmente como Educação Sanitária, surge no Brasil no início do século XX no período da República Velha onde o Estado brasileiro percebeu a necessidade de controlar as epidemias de doenças infectocontagiosas que abalavam a economia do país. Dentre os agravos se encontravam a tuberculose, varíola, febre amarela e sífilis, reflexo das péssimas condições sanitárias em que a população vivia. Dessa forma, surgiu a necessidade da mudança essencial dos hábitos da população e percebeu-se que a educação poderia ser uma forte aliada no combate não apenas a doenças infecciosas, mas das doenças crônico degenerativas, por isso, incentivar o amadurecimento do processo de educação em saúde e valorizar a saúde dentro da sua essência (ANDRADE LOM, 2001).

A partir de 1945, com o surgimento da Organização Mundial de Saúde (OMS), aumentou o debate sobre o processo saúde-doença, sendo definido como o estado de bem-estar mais completo, não se restringindo a simples ausência de doença. Apesar de esse conceito ser muito amplo e pouco definido, configurou-se na mudança do processo de educação sanitária (PELICIONI MCF, PELICIONI A, 2007).

Até meados da década de 70 , a educação em saúde era pautada em uma concepção higienicista, sanitarista, autoritarista, individualista e assistencialista, oriunda de normas e condutas de uma elite política e econômica que visava unicamente os seus interesses, mas com o passar do tempo observou-se que o processo de educação em saúde apresentava uma vertente que poderia respeitar todos os valores individuais com foco na subjetividade de cada individuo, assim como o papel do mesmo na sociedade além de observar também a ação das coletividades humanas (VASCONCELOS EM, 2006).

Após a modificação da visão da educação em saúde, a saúde passou a ser entendida como resultado da condição de vida da comunidade, influenciadas por fatores socioeconômicos, visando práticas que previnam as doenças e não apenas a cura. A educação em saúde, depois das mudanças dos ideais sanitários, passa a adquirir premissas capazes de desenvolver ações que modifiquem o comportamento e consequentemente melhore a qualidade de vida dos sujeitos (MACIEL MED, 2009).

Desta forma, a educação em saúde tornou-se um processo complexo que, ao unir um conjunto de saberes e práticas diversas, busca proporcionar às pessoas o mais alto nível de saúde. Esse conceito, entretanto, é fruto de um processo lento que vem ao longo dos anos acompanhando as mudanças políticas e econômicas no Brasil, bem como as diferentes concepções sobre a saúde e seus determinantes (SOUSA LB, et al., 2010).

A educação em saúde pode ser considerada uma das principais ações de promoção da saúde, já que se revela de suma importância tanto na prevenção e reabilitação de doenças, além de despertar a cidadania, responsabilidade pessoal e social, bem como a formação de multiplicadores e cuidadores. A educação em saúde está presente em todos os setores da atenção à saúde, desde atenção primária, centros de referência até a atenção hospitalar e é uma ação de impacto no cotidiano das pessoas e que representa 
uma intervenção de baixo custo que pode ser realizada não apenas por profissionais da saúde mas pelos diversos profissionais capacitados e habilitados para exercitar o processo educativo (FEIJÃO AR, GALVÃO MTG, 2007).

Desmembrando os termos educação e saúde, vê-se que ambos são igualmente importantes, complementam-se e funcionam como mecanismos interdependentes, já que mesmo com as transformações advindas dos progressos políticos, econômicos, sociais, eles coexistem, pois sem saúde não há educação, e sem educação não há saúde, assim acredita-se que o processo educacional e, principalmente, aquele relacionado à saúde está presente no cotidiano de todos os indivíduos e deve ser uma ação valorizada não apenas por profissionais da saúde, mas para todos aqueles que fazem parte dos grupos sociais e dos profissionais que agem diretamente ou indiretamente à valorização da saúde (COSTA R, PADILHA MI, 2012).

A educação em saúde caracteriza-se como uma forma extremamente direta e eficaz para a mudança de determinados comportamentos de risco, principalmente, por ser uma ação que pode atingir as necessidades sociais, mentais, biológicas e econômicas de uma população (CERVERA DPP, et al., 2011). Os profissionais e os grupos sociais, assim como as equipes de saúde, têm a corresponsabilidade de contribuir para a mediação entre os diferentes interesses, em relação à saúde, existentes na sociedade, porém as universidades assim como os cursos técnicos ainda possuem fragilidades no que condiz a ensino do processo educativo, além do fato que muitos profissionais ainda são formados dentro do conceito biomédico que valoriza o tratamento da doença ao invés da sua prevenção (CASARIN MR, PICCOLI JCE, 2010).

O estímulo a educação em saúde deve ser é uma ferramenta utilizada não apenas no ambiente universitário ou de cursos específicos na área da saúde, mas deve ser uma ferramenta valorizada desde a formação do ser humano na sua concepção de integrantes sociais, educar ainda é a forma mais barata de prevenção de doenças e representa um meio fundamental na redução de recursos financeiros, materiais e humanos no combate de doenças no mundo inteiro (CERVERA DPP, et al., 2011).

O estudo da educação em saúde justifica-se, pois representa ainda a forma mais barata de transformação de hábitos considerados deletérios à saúde, dessa forma, avaliar a perspectiva de alunos da área da saúde sobre tal tema representa avaliar o futuro da assistência em saúde deles. O estudo teve o objetivo de avaliar a representatividade do processo de educação em saúde entre estudantes da área da saúde.

\section{RELATO DE EXPERIÊNCIA}

Realizou no dia 4 de Dezembro de 2019, em uma Unidade Básica de Saúde localizada em Belém-Pará, uma roda de conversa com estudantes do $5^{0}$ semestre dos cursos de Graduação em Enfermagem, Fisioterapia e Nutrição, utilizou-se, primeiramente, a metodologia de observação da rotina assistencial do espaço de atenção em saúde para reflexão de como o processo de educação em saúde era efetivado no local.

Além disso, realizaram-se conversas informais com funcionários, usuários do serviço de saúde e familiares sobre os serviços ali prestados. Posteriormente, os alunos da área da saúde, que constituíam o grupo de estagiários da unidade relataram suas vivências e anseios no processo de atenção integralizada em saúde; o conceito de atenção holística se fez presente em 100\% dos estagiários presentes.

Os estagiários, dentro de sua totalidade (2 alunos de Enfermagem, 2 alunos de Fisioterapia e 2 alunos de Nutrição), expuseram suas perspectivas assistenciais e reconheceram o processo de educação em saúde como modelo válido para transformação dos atuais padrões de saúde que visam a terapia medicamentosa e valorização dos setores hospitalares, mas explanaram que a educação em saúde ainda precisa ser mais presente, não apenas no setor primário em saúde, mas em todos os setores assistenciais.

Percebeu-se que todos os estagiários ali presentes relataram que acreditam que o processo de educação em saúde seja um transformador na vida dos usuários, mas relataram também que o profissional responsável pelo processo educacional detém o conhecimento amplo responsável pela mudança dos 
paradigmas em saúde, sem considerar questões culturais e sociais previamente existentes, tal fato corrobora conceitos de que o educador é responsável por todo processo de modificação em saúde e que seu conhecimento é superior em relação aos fatores sócio culturais, modelo esse contraditório com as concepções de atenção holística.

Percebe-se que a concepção de magnitude do conhecimento ainda se faz presente entre muitos estudantes de saúde e que tal característica pode estar relacionada com a formação e transmissão de modelos que caracterizam o profissional de saúde como aquele detentor de todo conhecimento em saúde. Mudar a concepção da prioridade do tratamento medicamentoso ou até mesmo da visão biomédica não é fácil no atual contexto de formação, mas é necessário estimular a percepção da mudança de saúde dentro do ambiente educacional, ou seja, priorizar os comportamentos saudáveis para prevenir a doença propriamente instalada

\section{DISCUSSÃO}

Atualmente, faz-se necessário o retorno a educação em saúde como uma estratégia principal para o desenvolvimento do processo de produção de saúde que visa buscar a autonomia dos sujeitos e coletividades. Assim, a educação em saúde passa a ser desvinculada da doença e da prescrição de medicamentos e passa a ser considerada um método para a promoção da saúde, já que as informações e conhecimentos compartilhados farão com que os indivíduos desenvolvam atitudes e comportamentos para adotarem novos hábitos necessários para a prevenção de doenças e contatos com os agentes transmissores (BRASIL, 2008).

Porém, a educação em saúde ainda é um método desvalorizado por muito profissionais da saúde e outros envolvidos, para estimular a compreensão da importância desse processo e necessário estimular o pensamento holístico sobre o cuidado e assim permitir a interação de conhecimentos sem a idéia de que apenas um tipo de conhecimento é válido, mas adotar a compreensão de que os conhecimentos como um todo pode se unir e transformar as concepções tradicionais de atenção em saúde que por décadas ficaram enraizadas como sendo algo de direito apenas dos profissionais da saúde, no atual cenário brasileiro de atenção em saúde é possível observar a valorização da atenção terciária e é necessária uma reflexão para que outros setores, principalmente, a atenção primária possam ser mais valorizadas (COSTA R, PADILHA $\mathrm{Ml}, 2012)$.

Apesar desta nova visão, ainda há utilização do modelo tradicional de promover educação em saúde, conhecida como educação bancária, em que o educador "enche" os educandos de conteúdos, fazendo depósitos de comunicados. Neste modelo os sujeitos são vistos como seres passivos, de forma que cabe a educação e ao educador adaptá-los à realidade, considera-se que quanto mais se ensina mais se sabe, caracterizando que cabeça bem cheia implica no saber acumulado e empilhado (FIGUEIREDO MFS, et al., 2009). Entretanto, as práticas educativas visam o compartilhamento de conhecimento e reflexões críticas por parte dos sujeitos envolvidos proporcionando a busca de soluções de problemas relacionados à saúde (BRASIL, 2001).

A presença do educador com essa visão torna-se imprescindível e fundamental, pois é preciso que o saber seja extensivo a todos. Como um facilitador, o educador deve fornecer elementos para que o aluno e a comunidade se apropriem do conhecimento científico a respeito da saúde integral, considerando, ainda, o ser humano com sua subjetividade, emoções, sentimentos, pensamentos, desejos e necessidades, como também identificar e conhecer os fatores de risco determinantes do processo saúde-doença (COSTA R, PADILHA MI, 2012).

Sabe-se que para a realização do processo de educação em saúde o profissional não necessita apenas de técnica e/ou habilidade científica sobre o assunto, mas uma percepção diferenciada sobre o verdadeiro conceito de saúde que envolve o equilíbrio biológico, mental e social. Assim, considera-se que a educação em saúde deva ocorrer de maneira integral respeitando as reais necessidades dos indivíduos envolvidos focando os conhecimentos e saberes dos envolvidos (CERVERA DPP, et al., 2011). 
O educar é um processo contínuo que deve ser empregado no dia-a-dia. Deve-se, primeiramente, se conhecer para assim reconhecer e conhecer o outro e valorizar os conhecimento e vivências trazidas por esse usuário. A educação em saúde é considerada uma prática social articulada, que oferece possibilidade de partilhar conhecimentos políticos e sociais de forma ativa. É incluir o cidadão na ação como autor social, reflexivo e instrumentalizado com seu saber, contribuindo no processo de mudança social deste indivíduo (LEITE J, et al., 2010).

Alguns acadêmicos se aproximam do conceito da educação bancária, onde o profissional é o detentor do conhecimento científico e verdadeiramente correto sobre a saúde, e os usuários por outro lado, são aqueles que nada sabem e devem ser preenchidos com esse conhecimento, adquirindo dessa maneira, habilidades para manter uma qualidade de vida e saúde adequada. Observa-se tanto aqueles do curso de Enfermagem quanto Fisioterapia e Nutrição possuem o mesmo conceito, podendo inferir que o que está sendo repassado e ensinado refere-se a bases da educação bancária.

A educação em saúde ajuda o individuo a ter uma nova concepção de vida e ao mesmo tempo criar o comportamento de orientação ao próximo, assim cria-se um comportamento de transporte de informações agregando comportamentos saudáveis individuais e coletivos, levando em consideração todas as características biológicas, sociais e mentais do ser humano (COSTA R, PADILHA MI, 2012).

O novo panorama do cuidar necessita valorizar a promoção em saúde com a finalidade de não tornar o cuidado um simples ato de tratamento de doenças, mas é necessário evitar com que os indivíduos adoeçam, ou seja, a educação em saúde tem um papel fundamental na prevenção e pode significar uma redução significativa nos atuais gastos federais, estaduais e municipais no tratamento de patologias (CERVERA DPP, et al., 2011).

A necessidade da investigação dos grupos sociais por saberes e conhecimentos significa uma forma mais próxima de ofertar educação em saúde de qualidade, pois desse forma é possível ofertar um processo ético e igualitário, onde todos que fazem parte do processo podem somar a acrescentar de acordo com seu ambiente de vivência, assim é necessário investigar as reais necessidades da comunidade para que o processo de educação em saúde seja mais assertivo possível avaliando e intervindo nas reais necessidades dos grupos sociais e de cada indivíduo ali presente (COSTA R e PADILHA MI, 2012).

Desmembrando os termos educação e saúde, vê-se que ambos são igualmente importantes, complementam-se e funcionam como mecanismos interdependentes, já que mesmo com as transformações advindas dos progressos políticos, econômicos, sociais, eles coexistem, pois sem saúde não há educação, e sem educação não há saúde (LEITE J, et al., 2010).

A educação em saúde se apresenta como uma forma inovadora de aplicação em saúde que é contraditória em relação ao modelo tradicional biomédico que se baseia unicamente no tratamento de doença, mas instituir a educação em saúde na sociedade atual ainda não é tão fácil, pois a própria população acredita que formas terapêuticas mais eficientes ainda estão ligadas ao padrão biomédico e acreditam que o tratamento da doença é uma ação muito mais efetiva do que as ações preventivas previamente discutidas pelos órgãos em saúde (CERVERA DPP, et al., 2011).

Desta forma, a educação em saúde tornou-se um processo complexo que, ao unir um conjunto de saberes e práticas diversas, busca proporcionar às pessoas o mais alto nível de saúde. Esse conceito, entretanto, é fruto de um processo lento que vem ao longo dos anos acompanhando as mudanças políticas e econômicas no Brasil, bem como as diferentes concepções sobre a saúde e seus determinantes (CASARIN MR e PICCOLI JCE, 2010).

A educação em saúde permanece sendo um dos principais métodos de transformação em saúde e representa uma forma de baixo custo de intervenção no cuidado. Tal conceito de importância precisa se manter presente no cotidiano de estudantes e profissionais da saúde, assim, torna-se circunstancial a adoção de ações que possam valorizar o conceito de educação e de inserção desses estudantes/profissionais no âmbito do cuidado, a valorização das ferramentas de valorização da atenção básica, como as manobras de educação em saúde, são capazes de reduzir as dificuldades enfrentadas 
pelos serviços de saúde que representam a atenção secundária e primária, mas ainda é necessário conscientizar profissionais e estudantes sobre a responsabilidade que os mesmos possuem em efetivar as ações educacionais.

\section{REFERÊNCIAS}

1. ANDRADE LOM. SUS passo a passo: normas, gestão e financiamento. São Paulo: Hucitec; 2001.

2. BRASIL, Ministério da Saúde. Projeto Promoção da Saúde: Declaração de AlmaAta. Carta de Ottawa. Declaração de Adelaide. Declaração de Sundsvall. Declaração de Santafé de Bogotá. Declaração de Jacarta. Rede de Megapaíses e Declaração do México. Brasília, 2001.

3. BRASIL, Ministério da Saúde. Secretária de Gestão Estratégica e Participativa. Departamento de Apoio à Gestão Participativa. Bases para a Educação em Saúde nos Serviços. Brasília, 2008.

4. CASARIN MR, PICCOLI JCE. Educação em saúde para a prevenção do câncer de colo do útero em mulheres do município de Santo Ângelo/RS. Ciência e saúde coletiva, Rio de Janeiro, 2011; 16(9): 3925-32.

5. CERVERA DPP, et al. Educação em saúde: percepção dos enfermeiros da atenção básica em Uberaba (MG). Ciência e Saúde Coletiva, 2011; 16(1): 1547-54.

6. COSTA R, PADILHA MI. A unidade de terapia intensiva neonatal possibilitando novas práticas no cuidado ao recémnascido. Rev Gaúcha Enferm, 2012; 32(4): 32-4.

7. FEIJÃO AR, GALVÃO MTG. Ações de educação em saúde na atenção primária: revelando métodos, técnicas e base teórica. Revista. RENE, Fortaleza, 2007; 8(2): 41-49.

8. FIGUEIREDO MFS, et al. Educação em saúde no contexto da Saúde da Família na perspectiva do usuário. Interface Comunicação Saúde Educação, 2009; 16(41): 315-29.

9. LEITE J, et al. Educação em saúde: desafios para uma prática inovadora. 1a ed, São Caetano do Sul: Difusão Editora, 2010.

10. MACIEL MED. Educação em Saúde: conceitos e propósitos. Cogitare Enferm, 2009; 14(4): 773-6.

11. PELICIONI MCF, PELICIONI AF. Educação e promoção da saúde: uma retrospectiva histórica. O Mundo da Saúde, 2007; 31(3): 320-28.

12. SOUSA LB, et al. Práticas de Educação em Saúde no Brasil: a atuação da enfermagem. Rev Enferm UERJ, Rio de Janeiro, 2010; 18(1): 55-60.

13. VASCONCELOS EM. Educação popular e atenção à saúde da família. 3ª̣ ed. São Paulo: HUCITEC, 2006. 